\title{
NOWHERE SOLVABLE HOMOGENEOUS PARTIAL DIFFERENTIAL EQUATIONS ${ }^{1}$
}

\author{
BY HOWARD JACOBOWITZ AND FRANÇOIS TREVES
}

Nirenberg showed in [4] that the Lewy operator $L_{0}=\partial / \partial x+i \partial / \partial y-$ $2 i(x+i y) \partial / \partial u$ may be perturbed to obtain an operator $L_{1}$ such that if $L_{1} h=$ 0 in a connected neighborhood of the origin then $h$ is a constant in the neighborhood. In this note we eliminate the restriction to neighborhoods of a distinguished point and show that such operators are dense in the natural topology. We then announce a similar result for certain systems.

We consider operators defined on some open set $\Omega \subset \mathbf{R}^{3}$ where perhaps $\Omega$ is all of $\mathbf{R}^{3}$. Let $S=\left\{L=\sum_{j=1}^{3} \alpha_{j}(x) \partial / \partial x_{j} ; \alpha_{j} \in \mathbf{C}^{\infty}(\Omega, \mathbf{C})\right\}$. As the topology on $S$ we take the one induced by the usual topology on $\mathbf{C}^{\infty}(\Omega, \mathbf{C})$, namely uniform convergence of all derivatives on compact subsets. Note that this topology is metrizable and thus any countable intersection of open dense sets is dense.

DEFINITION. An operator $L \in S$ is aberrant if every $C^{\lambda}$ function $h$, with $\lambda>1$, satisfying $L h=0$ on some open connected subset of $\Omega$ is constant on that subset.

\section{THEOREM. The aberrant operators are dense in $S$.}

REMARKS. 1. The proof which follows is surprisingly simple and avoids the complicated construction in [4]. However Nirenberg's result only required that $h \in C^{1}$.

2. In our previous paper [1] we showed that any operator in $S$ with $L, \bar{L}$, and $[L, \bar{L}]$ linearly independent may be perturbed to an operator $L_{1}$ such that if $L_{1} h=0$ near the origin then $d h=0$ at the origin. As we shall see, a simple Baire category argument allows us both to conclude that $h$ is a constant and to eliminate the distinguished point.

3. The denseness holds in a finer topology since we work with only compactly supported perturbations. In particular we may find an aberrant operator $L$ for which $L, \bar{L}$, and $[L, \bar{L}]$ are linearly independent at each point of $\mathbf{R}^{3}$. Thus there exists a strictly pseudo-convex CR structure on $\mathbf{R}^{3}$ with only the constants as local CR functions.

We start the proof by defining a subspace of $S$. For each $P \in \Omega$ let $S_{P}=$ $\left\{L \in S\right.$; if $v \in C^{1}(\Omega)$ and $L v=0$ in a neighborhood of $P$, then $\left.d v(P)=0\right\}$.

Here $d v(P)$ represents the differential of $v$ acting on $T_{P} \mathbf{R}^{3}$.

LEMMA. For each $P \in \Omega, S_{P}$ is dense in $S$.

Received by the editors September 29, 1982 and, in revised form, January 18, 1983. 1980 Mathematics Subject Classification. Primary 35F05.

${ }^{1}$ This work partially supported by NSF Grants MCS- 8003048 and MCS-8102435. 
PROOF. Since this is an approximation result we may assume that $L$ is real analytic in some neighborhood of $P$ and that $L, \bar{L}$ and $[L, \bar{L}]$ are linearly independent there. We now follow the procedure used in [1]. Since $L$ is real analytic there exist solutions $L h_{1}=0$ and $L h_{2}=0$ with $d h_{1} \wedge d h_{2} \neq 0$ near $P$. If $F(z, w)$ is a holomorphic function then $h(x)=F\left(h_{1}(x), h_{2}(x)\right)$ also satisfies $L h=0$. Using this observation plus the fact that $L, \bar{L}$ and $[L, \bar{L}]$ are linearly independent we may introduce coordinates $(x, y, u)$ on $\Omega$ near $P$ such that $z=x+i y$ and $w=u+i G(x, y, u)$ are solutions of $L h=0$ and $G$ has the form $G=|z|^{2}+\alpha z u+\bar{\alpha} \bar{z} u+b u^{2}+\mathcal{O}\left((|z|+|u|)^{3}\right)$. In these coordinates $L=$ $\phi(x)\left(\left(1+i G_{u}\right) \partial / \partial \bar{z}-i G_{\bar{z}} \partial / \partial u\right)$ where $\phi(P) \neq 0$.

We consider the operator $R$ defined by $L=\phi R$. It suffices to find some $R_{1}$, defined in a neighborhood of the origin and uniformly close, along with its derivatives up to some large order, to $R$ in that neighborhood, with the property that if $v$ is $C^{1}$ and $R_{1} v=0$ near the origin, then $d v(0)=0$. For then we transform back to the original coordinates and extend $R_{1}$ appropriately to obtain our operator $L_{1}$.

Let $\Gamma(\lambda)=\{(z, u) ; w(z, \bar{z}, u)=\lambda\}$. Since $w=u+i G(x, y, u)$, with $G$ having the form given above, one can find some curve $\operatorname{Im} \lambda=\nu(\operatorname{Re} \lambda)$ passing through the origin in the $\lambda$-plane such that $\Gamma(\lambda)$ is a simple closed curve for $\operatorname{Im} \lambda>$ $\nu(\operatorname{Re} \lambda)$, a point for $\operatorname{Im} \lambda=\nu(\operatorname{Re} \lambda)$, and empty for $\operatorname{Im} \lambda<\nu(\operatorname{Re} \lambda)$. Let $\bar{\Omega}$ be a neighborhood of the origin symmetric with respect to the curves $\Gamma(\lambda)$ in the sense that whenever $\Gamma(\lambda) \cap \bar{\Omega}$ is nonempty, then $\Gamma(\lambda) \subset \bar{\Omega}$. Next let $\left\{C_{i}\right\}$ be a sequence of pairwise disjoint closed discs in the $\lambda$-plane, lying above the curve $\operatorname{Im} \lambda=\nu(\operatorname{Re} \lambda)$ and collapsing down to the origin. Let $T_{i}=$ $\left\{(z, u) ; w(z, \bar{z}, u) \in C_{i}\right\}$ be the corresponding closed topological solid torus. Now consider a function $h$ with $R h=0$ in $\bar{\Omega}-\bigcup T_{i}$. One may show that $\int_{\Gamma(\lambda)} h d z$ is a holomorphic function of $\lambda$ as long as $\Gamma(\lambda) \subset \bar{\Omega}-\bigcup T_{i}$. This holomorphic function becomes zero on the curve $\operatorname{Im} \lambda=\nu(\operatorname{Re} \lambda)$ and so is identically zero. It follows that $\iint_{\partial T_{i}} h d z \wedge d w=0$. But for any set with a smooth boundary

$$
\iint_{\partial T} h d z \wedge d w=\iiint_{T} d h \wedge d z \wedge d w=2 i \iiint_{T} R h d x d y d u .
$$

Now let $f_{1}(z, \bar{z}, u)$ be a $C^{\infty}$ function which is positive in each $T_{2 i+1}$ and zero everywhere else; similarly for $f_{2}$ and $T_{2 i}$. If $R h=f_{1} h_{u}+f_{2} h_{z}$ then

$$
\iiint_{T_{2 i+1}} f_{1} h_{u} d x d y d u=0 \text { and } \iiint_{T_{2 i}} f_{2} h_{z} d x d y d u=0 .
$$

It follows that $h_{u}(0)=h_{z}(0)=0$. Since $R h=0$ at the origin, one also has that $h_{\bar{z}}(0)=0$. Thus we take $R_{1}=R-f_{1} \partial / \partial u-f_{2} \partial / \partial z$ and use it, in the manner given above, to establish the lemma.

Our Baire category argument is a modification of that of Lewy [3]. Let $\left\{P_{j}\right\}$ be a countable dense set of points in $\Omega, N_{j}$ the open ball of radius $j^{-1}$ centered at $P_{j},\|h\|_{\lambda, j}$ the Hölder norm for $C^{\lambda}\left(N_{j}\right)$ and $\left|\omega\left(P_{j}\right)\right|$ any norm on the three dimensional vector space of co-vectors at $P_{j}$.

We let $E_{j, m, n}$ be the set of all those vector fields $L \in S$ for which there exists a function $h \in C^{1+1 / n}\left(N_{j}\right)$ such that 
1. $L h=0$ in $N_{j}$,

2. $\|h\|_{1+1 / n, j} \leq m$,

3. $\left|\operatorname{dh}\left(P_{j}\right)\right| \geq 1 / m$.

If $L \in$ closure $\left(E_{j, m, n}\right)$ then there exists some $h \in C^{1}\left(N_{j}\right)$ such that $L h=0$ in $N_{j}$ and $\left|d h\left(P_{j}\right)\right| \geq 1 / m$. (In fact more is true: $h \in C^{1+1 / n}$ and so $E_{j, m, n}$ is closed.) Thus $L \notin S_{P_{j}}$. Now the fact that $S_{P_{j}}$ is dense implies that $E_{j, m, n}$ is nowhere dense. By the Baire theorem there exists a dense set of vector fields none of which belongs to any $E_{j, m, n}$. Any one of these vector fields has the property asserted in the theorem.

To present a result for higher dimensions we consider complex vector fields $L_{1}, \ldots, L_{n}$ on $\Omega \subset \mathbf{R}^{2 n+1}$ with the following properties:

1. $L_{1}, \ldots, L_{n}, \bar{L}_{1}, \ldots, \bar{L}_{n}$ are linearly independent.

2. $\left[L_{j}, L_{k}\right]=0 \bmod \left\{L_{1}, \ldots, L_{n}\right\}$.

3. The Levi form has $n-1$ eigenvalues of one sign and one of the opposite sign.

Such vector fields form a space $\tau$ which we topologize using $C^{\infty}(\Omega, C)$. (It is of no concern that $\tau$ is not a linear space.)

It is a well-known result of Hörmander that under condition 3 (for $n>1$ ) any distribution which satisfies the system $L_{j} h=0$ is necessarily $C^{\infty}$. So we now say that the system of operators $L_{1}, \ldots, L_{n}$ is aberrant if every distribution solution to $L_{j} h=0$ for $j=1, \ldots, n$ on an open, connected subset of $\Omega$ is a constant function on that subset.

THEOREM. The aberrant operators are dense in $\tau$.

See [2] for the proof. Here we only note that the perturbation process in [1] for $n>1$ did not yield $d h=0$ and so a slightly more complicated procedure must be used. The difficulty, of course, is that one must only work with perturbations which preserve condition 2 (formal integrability).

\section{REFERENCES}

1. H. Jacobowitz and F. Treves, Non-realizable CR structures, Invent. Math. 66 (1982), 231-249.

2. __ Aberrant CR structures (volume in honor of T. Shirota, 1983), Hokkaido J. Math. (to appear).

3. H. Lewy, An example of a smooth linear partial differential equation without solution, Ann. of Math. (2) 66 (1957), 155-158.

4. L. Nirenberg, On a question of Hans Lewy, Russian Math. Surveys 29 (1974), 251-262.

Department of Pure and Applied Mathematics, Rutgers University, CAMDEN, NEW JERSEY 08102

Department of Mathematics, Rutgers University, New Brunswick, NEW JERSEY 08903 
